\title{
Spicing Up Dart with Side Effects
}

A set of extensions to the Dart programming language, designed to support asynchrony and generator functions

Erik Meijer, Applied Duality

Kevin Millikin, Google

Gilad Bracha, Google

The Dart programming language has recently incorporated a set of extensions designed to support asynchrony and generator functions. Because Dart is a language for Web programming, latency is an important concern. To avoid blocking, developers must make methods asynchronous when computing their results requires nontrivial time. Generator functions ease the task of computing iterable sequences.

The extensions described in this article provide synchronous functions in two flavors: normal functions that produce a single result; and generator functions that produce a sequence of results. It follows, for reasons of symmetry, that asynchronous functions should come in two flavors as well: those that produce a single result asynchronously; and generators that produce a sequence of results asynchronously. In the design described here, normal functions/generators and asynchrony/synchrony are orthogonal dimensions. Table 1 illustrates these dimensions and shows the types involved.

\begin{tabular}{|l|l|l|}
\hline & One & Many \\
\hline Sync & T & Iterable $<\mathrm{T}>$ \\
\hline Async & Future $<\mathrm{T}>$ & Stream $<\mathrm{T}>$ \\
\hline
\end{tabular}

TABLE 1 Generators and Synchrony: Orthogonal dimensions

Unfortunately, control structures in contemporary languages are optimized for simple synchronous calls, and as soon as the need arises to compose asynchronous methods, developers are left to their own devices, forced to write explicit CPS (continuation passing style) code by hand as if they were human compilers. Matters are even worse for code that manipulates asynchronous streams, but even creating synchronous iterables involves tedious manual construction of CPS state machines.

Fortunately, some modern language implementations already compile using continuations, which opens up the opportunity to perform the necessary CPS transformation automatically on the programmer's behalf. By simply marking method bodies as async, async*, or sync*, developers can write vanilla imperative control structures such as loops, conditionals, try/catch/finally, 
and break/continue statements to escape "callback hell" as the compiler takes care of all the heavy lifting.

\section{ASYNCHRONOUS PROGRAMMING USING FUTURES}

In Dart and many other languages, methods that asynchronously return a single result do this by immediately returning a value of type Future $\langle T\rangle$. The .then(f, onError: g) method on Future registers continuation callbacks for when the future completes successfully with a value $f$ of type $T$ or, respectively, with an exception $\mathrm{g}$. The reward for acknowledging asynchrony by using futures is "callback hell." Developers are not able to use regular control-flow constructs anymore and instead need to transform the code manually into CPS.

Imagine for a moment that Dart had a synchronous API for making HTTP requests. Then we could write the following straightforward code to create a new client, contact example.com to perform a search, print the result, and close the connection:

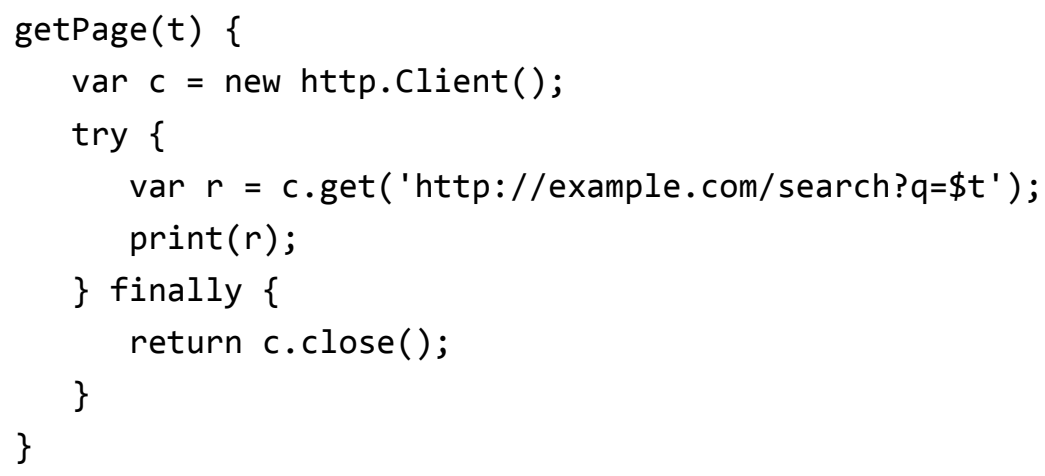


later completed in a way determined by the function body. If the function returns successfully, then the future is completed with the value computed by the function body. If the function throws an exception, then the future is completed with the object thrown.

With async functions, we can write fully asynchronous code, using regular control structures, very much like the imaginary blocking synchronous code we started with:

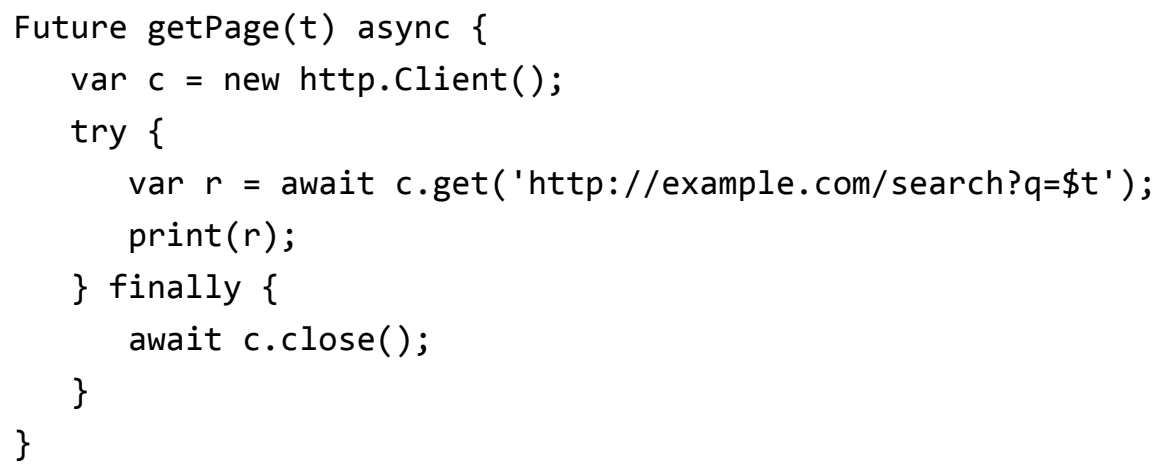

The async body shown here uses the await expression to suspend execution of the function until the future that is being awaited completes. Then, if the future completed with a value $r$, the await evaluates to $r$. If the expression that is awaited evaluates to a value that is not a future or throws an exception, the result is wrapped in a future before being processed further by the await.

Futures represent computations that eventually will complete with either a value or an exception. In Dart, we can register callbacks onValue(T value) and onError(Exception e) for those two events using the .then method on Future $\langle\mathrm{T}\rangle$. To create a future from scratch, Dart provides the factory type Completer $\langle$ T> (in other languages such as Scala, completers are often called promises). Given a completer, we can tear off a future via the .future property, and that future can be completed (at most once) with a value or an error via the . complete( $T$ value) or . completeError(Object e) methods.

Note that await e is just a normal expression inside an async function and thus can appear anywhere inside the body where an expression can appear, including inside catch and finally blocks. The async modifier can also be applied to the bodies of closures and getters but not on constructors or setters. As a result, an async function can be written in a way that is very close to how synchronous functions are written, with familiar sequential control constructs: loops, conditionals, and try-catch-finally. 
While async functions may remind us of the async/await feature in C\#, note that there are differences. In C\#, an async function executes synchronously from when it is called until the first await expression is encountered. Only if the value has not completed synchronously, or has thrown an exception, is the computation awaited. Otherwise, execution continues using the value of the completed future. As a result, an asynchronous function may be executed totally synchronously, even though marked as async. Another difference with C\# is that in Dart it is the function body that is marked async. This emphasizes that asynchrony is a property of the function's implementation, and the signature of a function is not affected. Furthermore, in Dart, await always suspends execution, even if the result of the expression being awaited is not a future. Finally, in C\# control cannot escape finally blocks, whereas in Dart, as in Java, finally blocks can transfer control back to wherever we want. This makes the C\# translation of async/await that retains the original program structure unfeasible in Dart, and this is what triggered the continuation-based implementation.

The async functions in Facebook's new Hack language are similar to C\#'s async methods and eagerly execute the body of an async function until the first await; already completed futures are not awaited.

Conceptually, the semantics of an await expression $\mathbf{x}=$ await e; rest $[\mathrm{x}]$ is e.then $((r)\{x=r$; rest $[x]\})$; of course, one of the subtleties is what exactly the "rest of the computation" means, especially when the await expression appears inside a try-catch-finally statement or a loop, as in the following (artificial) example:

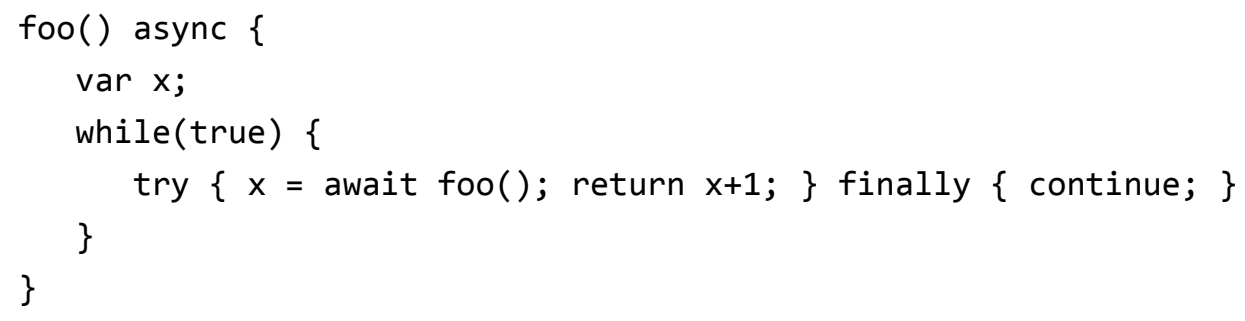




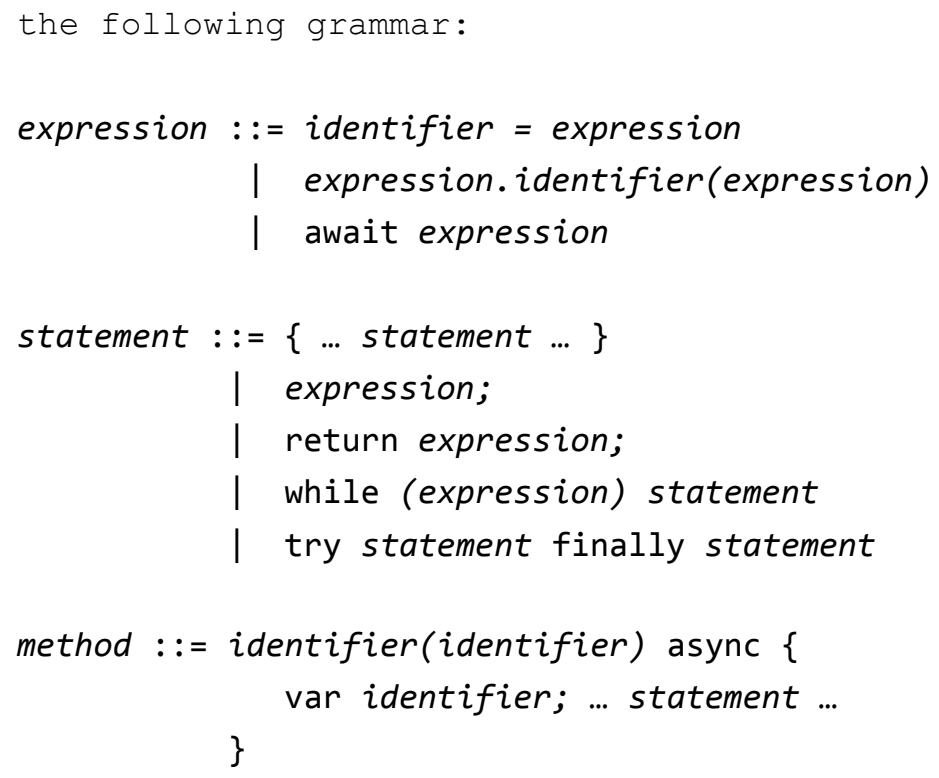

Without loss of generality, we assume that expressions and statements will always terminate successfully (i.e., no exceptions are thrown). Furthermore, to avoid clutter, we assume that methods have a single argument, method bodies have a single local variable, and all calls take a receiver and a single argument.

Don't be intimidated by the Greek symbols and higher-order functions. The semantic rules are based on the operational intuition of how Dart async methods are executed and match the declarative description of the official Dart language specification. ${ }^{2}$

The semantics for statements $\mathcal{C} \llbracket s \rrbracket(\rho, \sigma)$ takes two callback functions (or continuations) named $\rho$ and $\sigma$. The return continuation $\rho$ denotes what should happen if the statement $s$ executes return; the success continuation $\sigma$ denotes what should happen when execution of $\mathrm{s}$ falls off the end without encountering a return. Hence both continuations have the same type, i.e., they take any value and return void.

The semantics for expressions $\mathcal{E} \llbracket e \rrbracket(\sigma)$ needs only a success continuation $\sigma$ since return is a statement and hence one cannot return from a method inside an expression.

The semantics for method declarations $\mathcal{M} \llbracket d \rrbracket$ wires everything up by allocating a new Completer that is completed by the initial return and success continuations of the method body, and whose future is returned immediately by the generated wrapper function. The method body itself is run in a new future. Starting with the semantics of a return statement, the rule $\mathcal{C}$ 『return e;l $(\rho, \sigma)$ specifies that first the expression e is evaluated to obtain a value $r$, as indicated by the call $\mathcal{E} \llbracket \mathrm{e} \rrbracket(\lambda r \rightarrow .$.$) , and then the value of the expression$ 


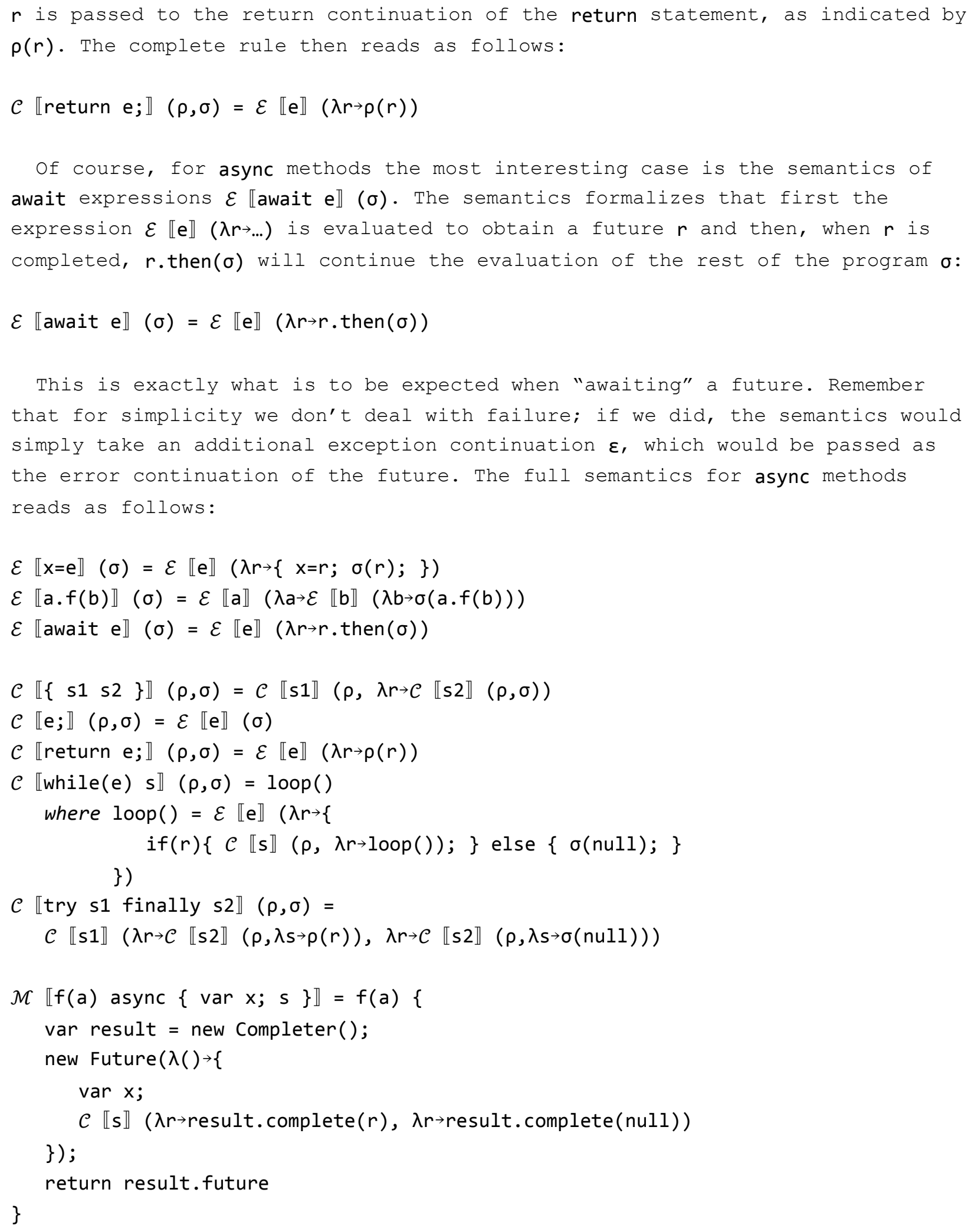




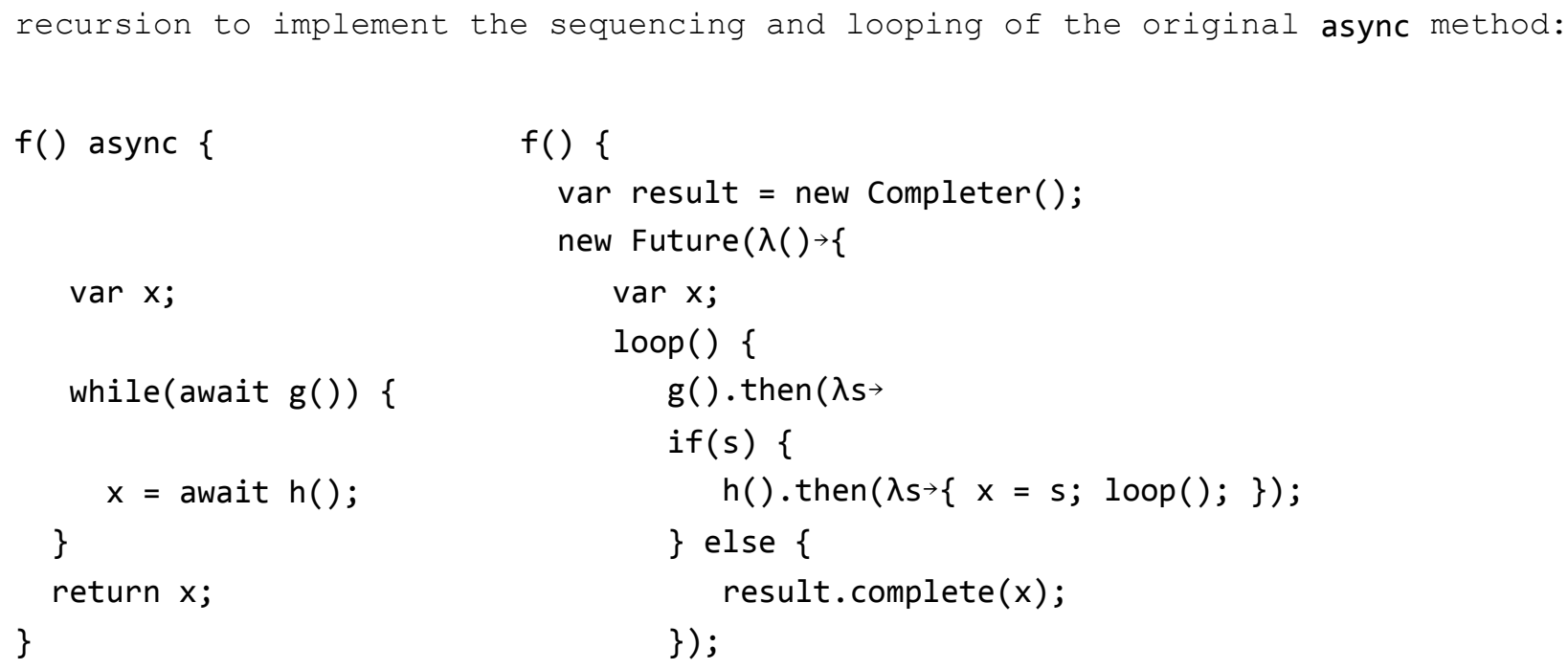

$f()$ async \{

$\operatorname{var} \mathbf{x}$;

while(await $g())\{$

$x=$ await $h()$;

\}

return $x$;

\}

A reassuring property of the abstract continuation semantics is that the resulting code is close to what a developer would have written by hand to achieve the same effect.

As noted, the semantics described here necessarily paints a simplified picture. To deal with the full Dart language and not just the featherweight subset, the Dart2Dart compiler carries around a couple of additional continuations to deal with break and continue statements, and switch and catch blocks. The compiler also defunctionalizes continuations back into direct style as much as possible (i.e., statements that contain no await expressions are compiled back down to themselves) and removes unnecessary "administrative" applications of continuations. Fundamentally, however, the compiler works the same as the semantics, except that it adds all the gory details required to move beyond the featherweight language category. To learn more about compiling with continuations, see the blog post by Matthew Might (http://matt.might.net/articles/cps-conversion/) and the references therein.

The Dart VM (virtual machine) has direct access to the stack, return addresses, and exception tables of the runtime and hence is able to implement the language enhancements described here at a lower level of abstraction. These runtime structures are concrete representations of the various continuations that the denotational semantics manipulates. To learn 
more about describing the semantics of async, sync*, and async* on the level

of stacks and return addresses, see the following papers:

http://dl.acm.org/citation.cfm?id=2367181 and

http://dl.acm.org/citation.cfm?id=1297063.

ITERATORS AND ITERABLES

Now that we have explained how async methods simplify asynchronous code that

returns a single value, let's shift the focus to bulk processing of

collections. The Iterable and Iterator interfaces (or slight variations

thereof) are the workhorses of collection libraries in almost every modern object-oriented language. Consuming iterables is deceptively easy because of the foreach loop:

Iterable $\mathrm{xs}=[1,2,3,4,5]$;

for $(x$ in $x s)\{\operatorname{print}(x)$; $\}$

What really happens is that for( $x$ in $x s$ ) hides the boilerplate of getting a fresh iterator from the iterable xs and iterating over that. In other words, a for loop in Dart is syntactic sugar for the following while loop:

Iterable xs $=[1,2,3,4,5]$;

var_ $\mathrm{xs}=\mathrm{xs}$.iterator;

while(_xs.moveNext()) $\{\operatorname{var} x=$ xs.current; print $(x)$; $\}$

Dart, however, has no syntactic support for producing iterables. This requires developers to perform the same cruel and unnatural acts that they had to do when writing asynchronous methods without the help of async functions. As an example, let's attempt to write the standard library function filter from scratch. Given a predicate, filter must return a new iterable where all values for which the predicate is false are removed:

\section{Iterable filter(Iterable src, predicate) \{ return new FilterIterable(src, predicate); \}}

The type FilterIterable builds on IterableBase, which implements all methods of Iterable except iterator. The iterator method returns an instance of Filteriterator that will filter out values from the iterator. 


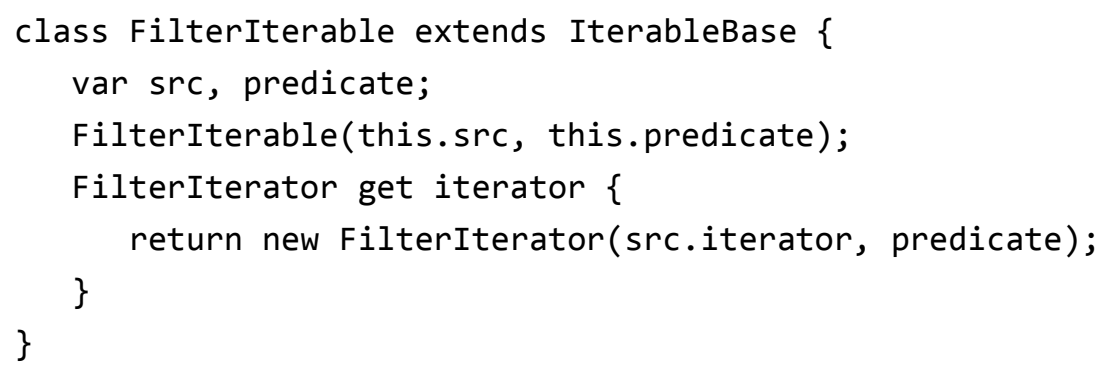

That is a lot of boring ceremony when the only interesting line is if(predicate(src.current))\{ ... produce the next value ... \}. It is a little harder to see at the moment that this code is actually also a manual CPS transform, but that will become clear in a moment, when the continuation semantics for synchronous generators is defined.

Synchronous generator functions are sugar for defining iterators and are defined by marking their body with the sync* modifier. Generators immediately return an Iterable when called. When we subsequently get an iterator from the iterable and call moveNext() on it, the body is executed until it hits a yield or return internally. If it reaches a return (again with the caveat it does not get hijacked by a finally clause), the iterator is done, and further calls to moveNext() will return false. Otherwise, subsequent calls to moveNext() resume the function where it left off, until the next yield or return. With generators, the code for filter collapses to a one-liner:

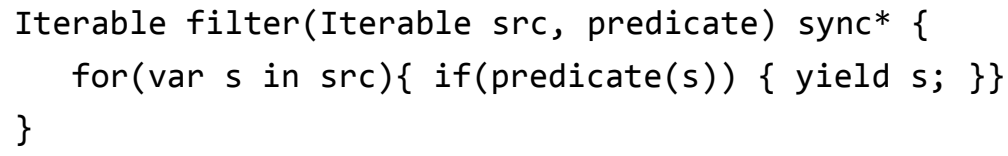


A careful reading of the API documentation for Future reveals that nested futures are automatically flattened. For generators we generally want to maintain nested iterables, but in certain cases we want to splice a nested iterable into its parent iterable. Take the following example where the sequence range $(s, n)=s, s+1, \ldots, s+n-1$ is recursively generated:

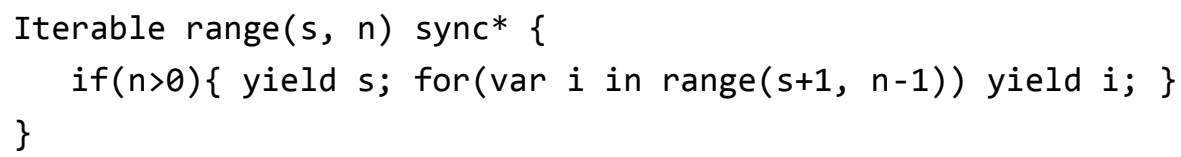

The problem with this implementation of range is that value $s+i$ is being yielded $i$ times, and hence the runtime complexity of range is quadratic. ${ }^{4}$ This is not an artificial problem; if we concatenate two iterables by copying

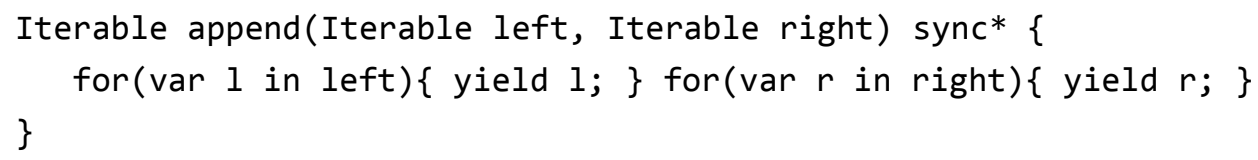

The ability to implement splicing of nested iterables efficiently requires the result of a sync* function to be a Rose-tree that is traversed in depthfirst order (using a stack) when we iterate over it. Formally, we use the fact that iterators are isomorphic to the recursive type of resumptions:

Iterator $\langle\mathrm{T}\rangle \cong \mu$ Resumption .

()$\rightarrow(() \cup(T$, Resumption $) \cup(() \rightarrow$ Resumption, Resumption $))$

A Resumption is a recursive function that unfolds an iterator either by terminating immediately, by returning a pair of a single value and a continuation resumption that will produce more values, or by returning a pair 


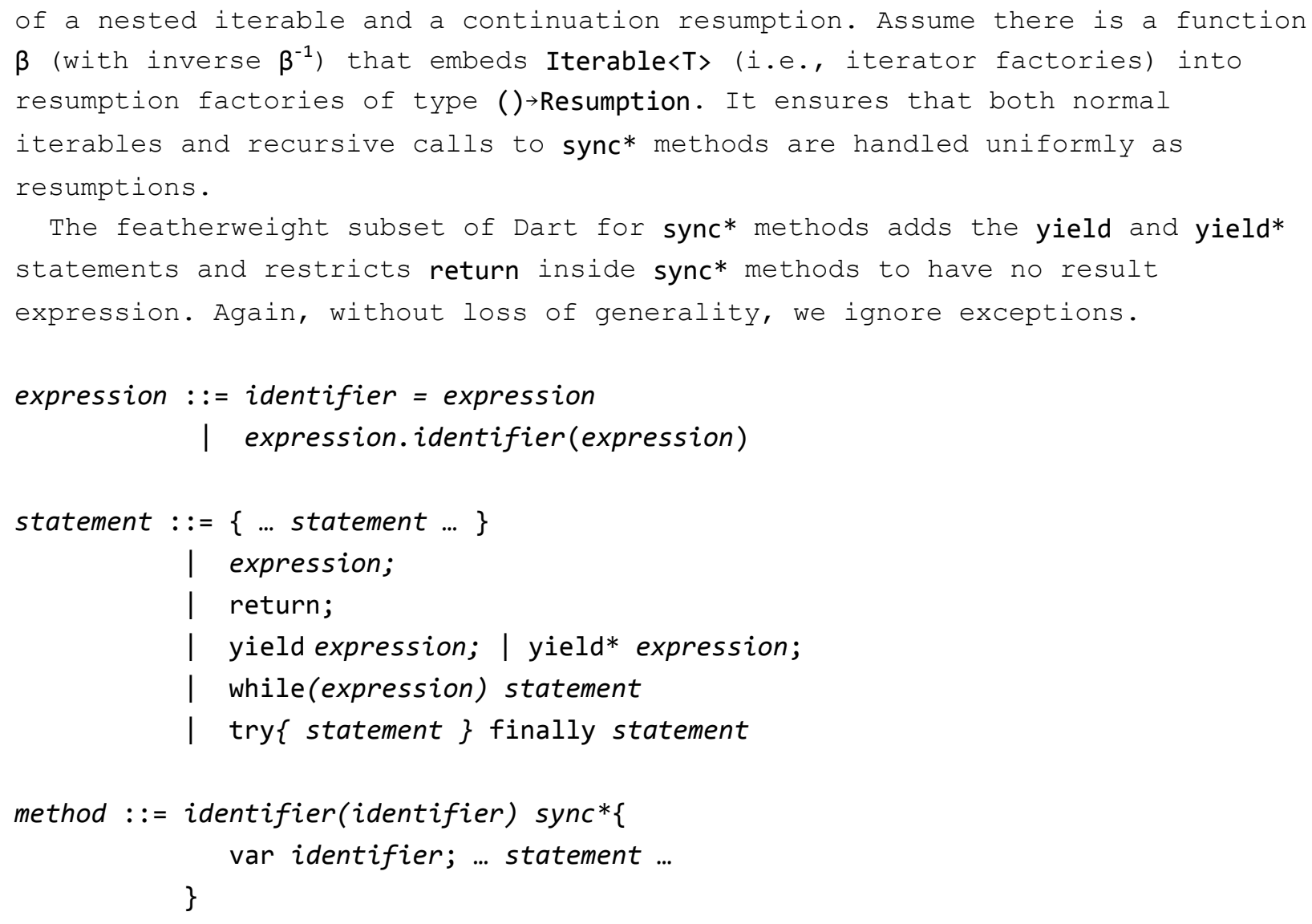

The semantics for statements $\mathcal{C} \llbracket s \rrbracket(\rho, \sigma)$ uses two continuations $\rho$ and $\sigma$, since when executing sync* bodies we need to run finally blocks before completing the underlying iterable. The semantics for expressions $\mathcal{E} \llbracket \mathbf{e} \rrbracket(\sigma)$ remains pretty much as for async methods except that inside a sync* method we cannot await (for that we need async* methods, as explained later in this article). The semantics for method declarations $\mathcal{M} \llbracket d \rrbracket$ wires everything up.

Return statements inside sync* methods don't have a result expression but have to go through the finally blocks like return statements in normal methods; hence, the semantics of a return statement $\mathcal{C} \llbracket$ return;》 $(\rho, \sigma)$ invokes the return continuation with an "end of resumption" value:

$\mathcal{C} \llbracket$ return; $\rrbracket(\rho, \sigma)=\rho()$

For sync* methods the most interesting cases yield a single value $\mathcal{C}$ 【yield e;】 or a nested iterable $\mathcal{C} \llbracket y i e l d^{*}$ e;》. The semantics formalizes that first the expression $\mathcal{E} \llbracket \mathbf{e} \rrbracket(\lambda r \rightarrow \ldots)$ is evaluated to obtain a result $r$, and then the execution of the sync* method returns a resumption pair of the value $r$ and the rest of the computation $\sigma$ :

$\mathcal{C} \llbracket$ yield $\mathrm{e} ; \rrbracket(\rho, \sigma)=\mathcal{E} \llbracket e \rrbracket(\lambda r \rightarrow(r, \sigma)))$ 
When we yield* a nested iterable, $r$ is coerced to a resumption using $\beta$ so we do not have to worry about the difference between a recursive invocation of a sync* method or a regular method that returns an iterable.

$\mathcal{C} \llbracket y$ yield* e; $(\rho, \sigma)=\mathcal{E} \llbracket e \rrbracket(\lambda r \rightarrow(\beta(r), \sigma))$

The full continuation semantics for sync* methods then reads as follows:

$\mathcal{E} \llbracket \mathrm{x}=\mathrm{e} \rrbracket(\sigma)=\mathcal{E} \llbracket \mathrm{e} \rrbracket(\lambda r \rightarrow\{\mathrm{x}=\mathrm{r} ; \sigma(r) ;\})$

$\mathcal{E} \llbracket a . f(b) \rrbracket(\sigma)=\mathcal{E} \llbracket a \rrbracket(\lambda a \rightarrow \mathcal{E} \llbracket b \rrbracket(\lambda b \rightarrow \sigma(a . f(b)))$

$\mathcal{C} \llbracket s 1 s 2 \rrbracket(\rho, \sigma)=\mathcal{C} \llbracket s 1 \rrbracket(\rho, \lambda r \rightarrow \mathcal{C} \llbracket s 2 \rrbracket(\rho, \sigma))$

$\mathcal{C} \llbracket e ; \rrbracket(\rho, \sigma)=\mathcal{E} \llbracket e \rrbracket(\lambda r \rightarrow \rho(r))$

$\mathcal{C} \llbracket$ return; $\rrbracket(\rho, \sigma)=\rho()$

$\mathcal{C} \llbracket y i e l d \mathrm{e} ; \rrbracket(\rho, \sigma)=\mathcal{E} \llbracket \mathrm{e} \rrbracket(\lambda r \rightarrow(r, \sigma))$

$\mathcal{C} \llbracket$ yield* e;》 $(\rho, \sigma)=\mathcal{E} \llbracket e \rrbracket(\lambda r \rightarrow(\beta(r), \sigma))$

$\mathcal{C} \llbracket$ while(e) $s \rrbracket(\rho, \sigma)=\operatorname{loop}()$

where $\operatorname{loop}()=\varepsilon \llbracket e \rrbracket(\lambda r \rightarrow\{$

$\operatorname{if}(r)\{\mathcal{C} \llbracket s \rrbracket(\rho, \lambda r \rightarrow \operatorname{loop}()) ;\}$ else $\{\sigma(n u l l) ;\}$

\})

$\mathcal{C} \llbracket$ try $s 1$ finally $s 2 \rrbracket(\rho, \sigma)=$

$\mathcal{C} \llbracket s 1 \rrbracket(\lambda() \rightarrow \mathcal{C} \llbracket s 2 \rrbracket(\rho, \lambda s \rightarrow \rho()), \lambda r \rightarrow \mathcal{C} \llbracket s 2 \rrbracket(\rho, \lambda s \rightarrow \sigma(r)))$

$\mathcal{M} \llbracket f(a)$ sync* $\{\operatorname{var} x ; s\} \rrbracket=f\left(\_a\right)\{$

return new_IterableBase $(\lambda() \rightarrow\{$

$\operatorname{var} \mathrm{x}$; var $\mathrm{a}=\ldots \mathrm{a}$; return $\mathcal{C} \llbracket s \rrbracket(\lambda() \rightarrow(), \lambda r \rightarrow())$

\});

\}

where

class_IterableBase extends IterableBase \{

var_resumption;

_IterableBase(this._resumption);

Iterator get iterator $\left\{\right.$ return $\beta^{-1}$ (_resumption()); \}

\} 


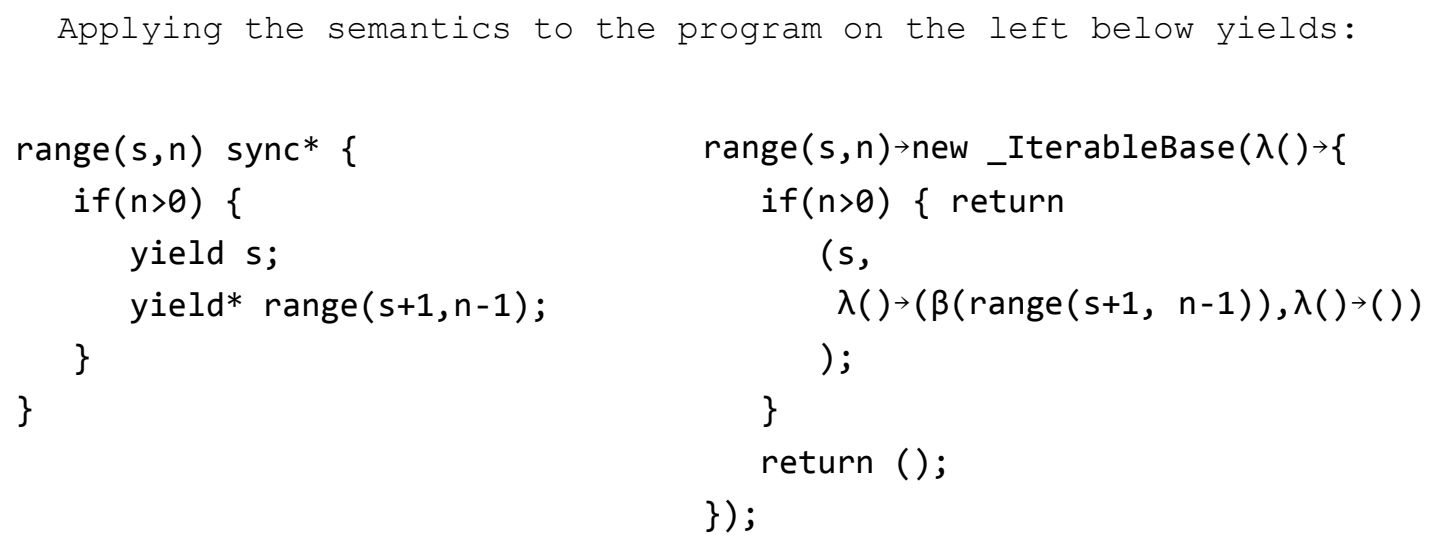

\section{To Cancel or Not To Cancel Is the Question}

An open design decision is whether or not Dart iterators should support a cancel or dispose method. For simple iterators that is no problem, but once the power of sync* methods is introduced, it is easy to leave a method hanging in-flight holding on to expensive resources. For example, calling moveNext() just once on the following sync* method will return the first line of the file but never close it:

Iterable readLines(name) sync* \{

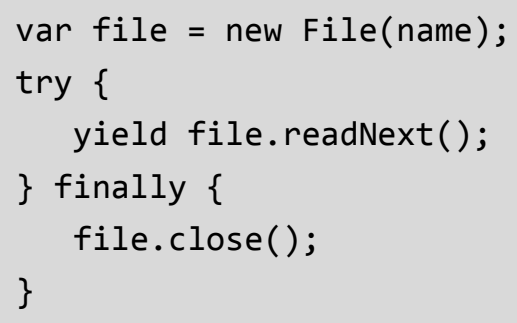


In C\# when we dispose (cancel) an iterable, all finally blocks are run, and the foreach loop is surrounded by a try/finally block to ensure that the enumerable is always disposed of after use. Adding this feature to sync* methods can be easily achieved by maintaining a "finally continuation" that when invoked will run all finally blocks and ignore any further yield and yield* statements encountered during cleanup.

ASYNC* $=$ ASYNC+SYNC*

So far, we have seen how async and sync* methods simplify coding of methods that asynchronously return a single value and methods that synchronously return multiple values, respectively. Combining these two dimensions results in async* functions that simplify coding of methods that asynchronously produce streams of values. Just as an async function immediately returns a future, an async* function immediately returns a stream. When (and only when) we listen to the stream, the function starts executing, just as a sync* method starts execution when moveNext is called on the Iterable it returns.

Say we want to scan a stream and incrementally emit the intermediate results of applying an asynchronous function to each incoming item. Using async* methods, this becomes just a matter of looping over the stream, awaiting the computation of the next state, and then yielding the current state:

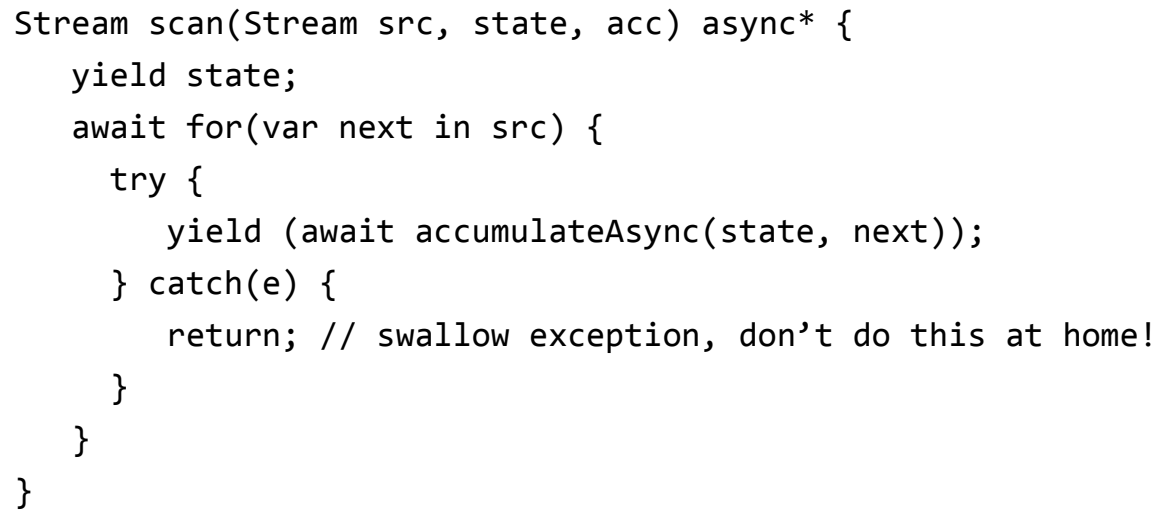

As you can imagine, manually implementing this behavior using a StreamController is very tedious. We need to pause the source stream while awaiting the result of accumulateAsync and resume it when the future completes, and we need to handle a try-catch block around the await, cancellation of the resulting stream, etc. Thanks to async* methods, we can just write regular control-flow, and the Dart compiler will take care of handling all the difficult stream-management issues. 
Dart streams are the asynchronous counterparts of iterables. Given a stream ts of type Stream $\langle T\rangle$, we can listen to the stream for notification of new values by passing a callback void onData( $T$ event), and optionally void onError(Exception e) and void onDone(). The listen method then returns a StreamSubscription that has a method cancel to unsubscribe from the stream, plus pause and resume to control the speed of the producer. Streams are created either by transforming existing streams using operators such as map and reduce or by using a StreamController to which we can push values using the methods add and addError.

The featherweight subset of Dart that defines the semantics for async* methods is defined as follows:

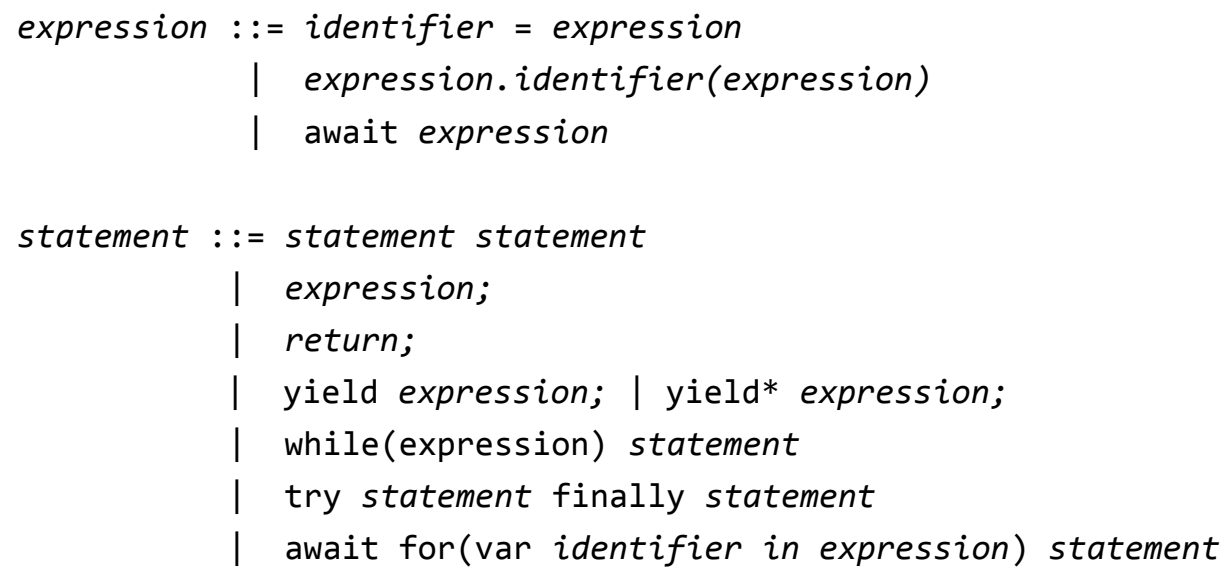

To keeps things simple, we will again ignore failure and not implement cancellation. Because await for loops can be nested, the semantics for statements in a featherweight async* body $\mathcal{C} \llbracket s \rrbracket(\xi, \rho, \sigma)$ takes not only two continuations $\rho$ and $\sigma$, but also a subscription $\xi$ to the stream of the enclosing method or await for loop (for convenience, we often refer to $\xi$ simply as "the immediately enclosing stream"). The immediately enclosing stream is needed so that when the result stream of the async* method is paused, we can pause/resume the body at a yield or yield* statement or when iterating over a nested stream using await for. The enclosing stream is initialized with never, which never emits a value, and hence never.pause() and never.resume() are no-ops and can be dropped. 
The semantics for expressions $\mathcal{E} \llbracket \mathbf{e} \rrbracket(\xi, \sigma)$ also gets the subscription to the enclosing stream so it can pause/resume it when executing an await expression. The semantics for method declarations $\mathcal{M} \llbracket d \rrbracket$ wires everything up by creating a subclass of stream controller whose . stream is returned immediately and whose .sink is used by the body of the async* method to yield values.

For expressions $\mathcal{E}$ 【await $\mathrm{e} \rrbracket(\xi, \sigma)$, first evaluate the expression

$\mathcal{E} \llbracket \mathbf{e} \rrbracket(\xi, \lambda r \rightarrow\{\ldots\}\}$ using the enclosing stream $\xi$ to yield a value $r$. Before awaiting $r$, pause $\xi$ so that no values are pushed while waiting for the future to complete. Before the success continuation is invoked with the result $\mathbf{v}$ of the future, resume the enclosing stream $\xi$ :

$\mathcal{E} \llbracket$ await $\mathrm{e} \rrbracket(\xi, \sigma)=\mathcal{E} \llbracket \mathrm{e} \rrbracket(\xi, \lambda r \rightarrow\{$

$\xi$.pause ()$; r$.then $(\lambda v \rightarrow\{\xi$. resume ()$; \sigma(v) ;\})$;

\})

Return statements inside async* methods are similar to sync* returns in that they invoke the return continuation to run all outstanding finally blocks and then close the result stream. Before yielding values, an async* method checks if its result stream, captured in the global variable result, has a pending pause request. If so, the immediately enclosing stream $\xi$ is paused, then the result stream itself is paused by setting the onResume callback to continue when it is resumed by the consumer:

$\mathcal{C} \llbracket$ yield e; $(\xi, \rho, \sigma)=\mathcal{E} \llbracket e \rrbracket(\xi, \lambda r \rightarrow\{$

if(result.isPaused) \{ $\xi$.pause () ; result._onResume $=\lambda() \rightarrow\{$ $\xi . r e s u m e()$; result.add $(r) ; \sigma(n u l l)$;

\} ;

\} else \{

result.add $(r) ; \sigma(n u l l)$;

\}

\})

A similar pause check is performed before closing the result stream in the initial return and success continuation when $\mathcal{M} \llbracket \rrbracket$ is invoked on the async* body. The most interesting case for async* methods is iterating over a stream $\mathcal{C} \llbracket$ await for $(\operatorname{var} \mathrm{x}$ in e) $\{\mathrm{s}\} \rrbracket(\xi, \rho, \sigma)$. First evaluate the loop expression $\mathcal{E} \llbracket \mathbf{e} \rrbracket(\xi, \lambda r \rightarrow\{\ldots\})$ to obtain a stream $r$. Then pause the immediately enclosing stream $\xi$ and listen to $r$ instead to get the subscription _ $\xi$. The onData callback executes the loop body in the context of _ $\xi$, and the onDone callback restores $\xi$ before invoking the success continuation: 


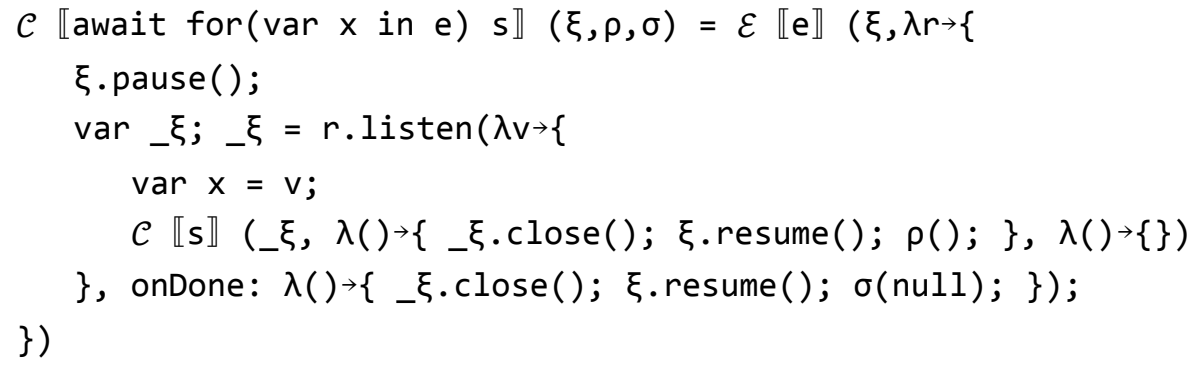




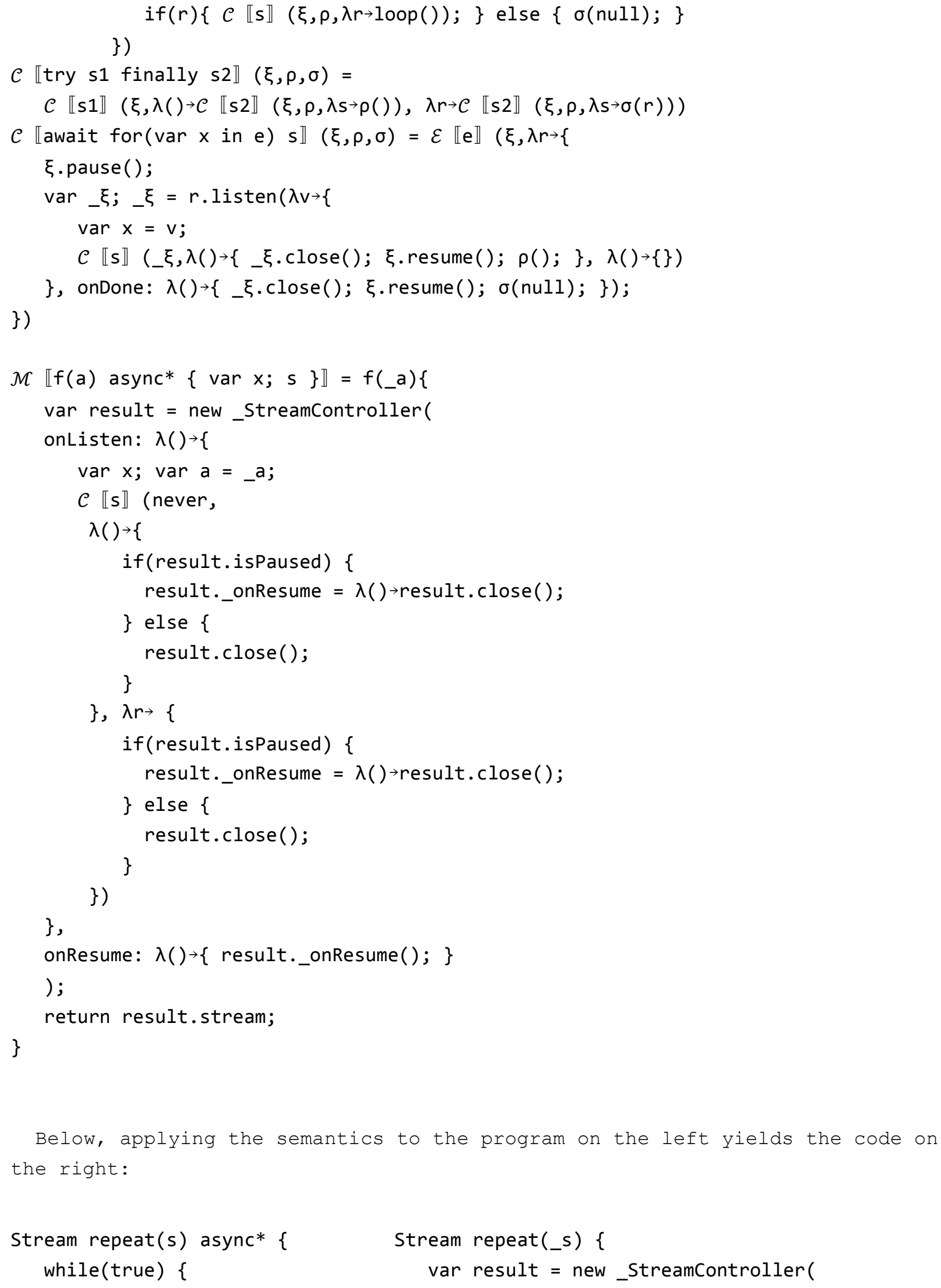


asynchronous computations, as well as for producing synchronous and

asynchronous streams of values. In the near future, every contemporary programming language will likely support asynchronous methods in one way or another, and the semantics used in this article may aid other language designers in adopting the approach to fit the idiosyncrasies of their particular languages.

\section{REFERENCES}

1. Bonér, J. 2012. Latency numbers every programmer should know; https://gist.github.com/jboner/2841832.

2. Dart Language Specification; https://www.dartlang.org/docs/spec/. 3. Hughes, R. J. M. 1986. A novel representation of lists and its application to the function "reverse"; Information Processing Letters 22 (3): 141-144; http://www.cs.tufts.edu/ nr/cs257/archive/john-hughes/lists.pdf.

4. Jacobs, B., Meijer, E., Piessens, F., Schulte, W. Iterators revisited: proof rules and implementation; http://research.microsoft.com/enus/projects/specsharp/iterators.pdf.

5. Stoy, J. E. 1977. Denotational Semantics: The Scott-Strachey Approach to Programming Language Theory. Cambridge, MA: MIT Press;

http://dl.acm.org/citation.cfm?id=540155.

\section{LOVE IT, HATE IT? LET US KNOW} FEEDBACK@QUEUE . ACM . ORG

ERIK MEIJER (emeijereapplied-duality.com) is the founder of Applied Duality and professor of big-data engineering at TUDelft. He is perhaps best known for his contributions to programming languages such as Haskell, C\#, Visual Basic, Hack, and Dart, as well as his work on big-data technologies such as LINQ and the Rx Framework.

KEVIN MILLIKIN (kmillikin@google.com) is a software engineer at Google who has worked on the V8 JavaScript engine and the Dart virtual machine. He was one of the original developers of V8's Crankshaft compiler, the first JavaScript compiler to feature adaptive optimization. He is currently working on dart2js. GILAD BRACHA (gbracha@google.com) is the creator of the Newspeak (http://bracha.org/Site/Newspeak.html) programming language and coauthor of the Java Language Specification (http://docs.oracle.com/javase/specs/). He is currently a software engineer at Google where he works on Dart (https://www.dartlang.org/).

(c) 2014 ACM 1542-7730/14/1200\$10.00 